\section{Jourant of Vascular}

Aardenburg, R. 350

Abe, K. 123

Abegunewardene, N. 45

Agabiti-Rosei, E. 512

Ai, J. 402

Aihara, Y. 402

Alexis, J.D. 538

Al Haj Zen, A. 529

Angelini, G.D. 521

Angus, J.A. 279

Aoki, T. 343

Ares, M.P.S. 395

Asahara, T. 437

Au Yeung, K. 314

Ažman-Juvan, K. 164

Bakker, E.N.T.P. 10, 271

Barbour, J.R. 457

Bauriedel, G. 173

Becker, D. 45

Beissert, S. 103

Bengtsson, E. 395

Bény, J.-L. 416

Beretz, A. 386

Berges, A. 480

Bernjak, A. 164

Bierbach, B. 45

Birch, D.J. 323

Birnbaum, D.E. 333

Bittinger, F. 45

Boari, G.E.M. 512

Bolitho, C. 193

Bond, M. 521

Booth, A.D. 357

Bouges, S. 457

Boulos, P.B. 323

Boumans, M.-L. 427

Bouwman, F.G. 427

Brandenburg, V.M. 427

Breier, G. 437

Breithardt, G. 103

Brismar, K. 295

Brozovich, F.V. 445

Bruneval, P. 529

Burnstock, G. 323

Busser, E. 244

Charo, I.F. 538

Chen, F.C. 445
Chen, P.-L. 111

Chereshnev, I. 538

Choi, W.S. 259

Chung, A.W. 357

Chung, A.W.Y. 314

Cloutier, I. 503

Colantuoni, A. 69, 89

Colliec-Jouault, S. 529

Cranenburg, E.C.M. 427

Crevel, H. 181

Dardik, A. 251

Dear, A.E. 143

De Ciuceis, C. 512

Del Soldato, P. 521

Demcheva, M. 222

De Mey, J.G.R. 350

de Smet, B.J.G.L. 493

de Weger, R.A. 244

Dias, N. 395

Doevendans, P.A. 244

Dousha, L.F. 143

Dujols, C. 529

Durand, E. 529

Dwarakanath, R.S. 132

Easton, A. 111

Eldridge, J. 222

Eschert, H. 103

Essl, C. 437

Etienne-Selloum, N. 386

Ettelaie, C. 19

Fancher, T.T. 251

Fernandes e Fernandes, J. 395

Fischer, A.-M. 529

Fitzgerald, T.N. 251

Fredrikson, G.N. 395

Furukawa, H. 233

Gaspari, T.A. 143

Geoffroy, P. 503

Geraldes, P. 503

Gilbert, T.R. 205

Girolamo, F. 129

Giulini, S.M. 512

Gloe, T. 153

Gonçalves, I. 395

Goris, M. 493
Goumans, M.-J. 244

Grant, S. 375

Grau, G.E. 78

Groenendijk, B.C.W. 54

Groenewegen, H.C. 493

Groesser, L. 153

Guibert, C. 181

Hackeng, T.-M. 427

Hamamoto, A. 233

Harada, N. 233

Hara-Kaonga, B. 1

Hardeman, M.R. 10

Harrington, A. 1

Hashimoto, N. 343

Hattori, A. 233

Heaton, N.S. 365

Heidenreich, R. 437

Helley, D. 529

Hellwig, N. 153

Hierck, B.P. 54

Hillebrands, J.-L. 493

Hoch, J.R. 365

Horstick, G. 45

Hosaka, T. 233

Houamed, K.M. 402

Hullett, D.A. 365

Humboldt, T. 480

Hyodo, F. 123

Ikonomidis, J.S. 457

Ikuta, K. 123

Ishikawa, M. 343

Jabs, A. 173

Jahromi, B.S. 402

Janssen, G.M.J. 350

Jeremy, J.Y. 521

Johnson, M.J. 1

Jones, J.A. 457

Jörneskog, G. 295

Jovinge, S. 395

Kalani, M. 295

Kane, M.O. 386

Karger, T. IV

Kataoka, H. 343

Katayama, Y. 123

Kato, K. 343
Katz, J. 538

Kawano, T. 233

Kawarazaki, S. 343

Kempski, O. 45

Ketteler, M. 427

Kim, B. 259

Kim, H.J. 259

Kita-Matsuo, H. 343

Koenigsberger, M. 416

Konstantinides, S. 480

Koos, R. 427

Koyama, K. 233

Kraemer, S. 173

Krötz, F. 153

Kunz-Schughart, L.A. 333

Kusumi, Y. 303

Kwaijtaal, M. 427

Lafont, A. 529

Lamers, D. 244

Lanting, L. 132

Lapi, D. 69, 89

Lauterbach, M. 45

Lebrun, S. 480

Lee, C.-K. 259

Lee, H.M. 259

Lehle, K. 333

Lehr, H.-A. 45

Leifheit, M. 480

Levin, A. 357

Li, Y. 205

Liaw, L. 1

Lindken, R. 54

Liu, H.B. 143

Liu, Q.-H. 469

Lou, J.N. 78

Macdonald, R.L. 402

Malinowski, R.L. 365

Maloney, S.P. 251

Mannell, H. 153

Marchiafava, P.L. 69, 89

Marthan, R. 181

Martinez-Lemus, L.A. 211

Matsumoto, A.H. 205

Mawatari, K. 233

Meister, J.-J. 416

Meurrens, K. 480

Miceli-Libby, L. 1 
Miclini, M. 512

Miller, M.R. 375

Mitsumata, M. 303

Moberg, A. 395

Moll, F.L. 244

Montan, J. 395

Moriwaki, T. 343

Morizumi, R. 233

Moses, J. 395

Muise-Helmericks, R.C. 222

Mulvany, M. 547

Murayama, T. 437

Muto, A. 251

Muzaffar, S. 521

Nakamura, A. 233

Nakano, M. 233

Nakaya, Y. 233

Natarajan, R. 132

Newby, A.C. 521

Ng, A.-K. 1

Nickenig, G. 173

Nieuwstadt, F.T.M. 54

Nikitina, E. 402

Nilsson, J. 395

Niu, J.X. 78

Nozaki, K. 343

Oi, K. 123

Onuta, G. 493

Oshita, S. 233

Osol, G. 547

Ozerdem, U. 129

Paiardi, S. 512

Park, P.-J. 259

Park, S.H. 259

Park, T.-K. 259

Pasterkamp, G. 244

Pedro, L.M. 395

Peeters, L.L.H. 350

Peeters, W. 244

Pernow, J. 295

Perris, R. 129

Pimiento, J.M. 251

Pistea, A. 10, 271

Plank, C. 153
Platto, C. 512

Poelmann, R.E. 54

Pohl, U. 153

Porteri, E. 512

Post, S. 244

Pradier, A. 19

Preuner, J.G. 333

Pyo, R.T. 538

Rathore, R. 469

Reddy, M.A. 132

Rizzardi, N. 512

Rizzoni, D. 512

Robenek, H. 103

Röcken, M. 437

Rodat-Despoix, L. 181

Roks, A.J.M. 493

Rollins, B.J. 538

Roncali, L. 129

Rose, C. 357

Ross, G.R. 33

Rozing, J. 493

Rupprecht, L. 333

Sadamasa, N. 343

Sahar, S. 132

Saito, S. 343

Sarr, M. 386

Sauser, R. 416

Savineau, J.-P. 181

Sawalich, M. 480

Schaefer, K. 480

Scheld, H.H. 103

Schini-Kerth, V.B. 386

Schleef, R. 480

Schlindwein, P. 45

Schmid, C. 333

Schreml, S. 333

Schroeter, M.R. 480

Schurgers, L.J. 427

Seppey, D. 416

Settergren, M. 295

Shi, W. 205

Shimokawa, H. 123

Shukla, N. 521

Silver, M. 437

Sindermann, J.R. 103
Sirois, M.G. 503

Skowasch, D. 173

Sluijter, J.P.G. 244

Sohn, H.-Y. 153

Song, H. 259

Spaan, J.A.E. 10

Spaanderman, M.E.A. 350

Sparatore, A. 521

Spinale, F.G. 457

Štajer, D. 164

Stallcup, W.B. 129

Stefanovska, A. 164

Stekelenburg-de Vos, S. 54

Stemerman, M.B. 132

Stephens, S.L. 457

Stroud, R.E. 457

Sugimura, K. 123

Takagi, Y. 343

Takahashi, A. 233

Tang, P.C.Y. 251

Tanguay, J.-F. 503

Taubman, M.B. 538

Tellides, G. 251

Thompson, C.R. 357

Tiberio, G.A.M. 512

To, F. 395

Turmaine, M. 323

Urbančič-Rovan, V. 164

Ursem, N.T.C. 54

Utsumi, H. 123

Uwatoku, T. 123

VanBavel, E. 10, 271

van Breemen, C. 314, 357

van Eijndhoven, H.W.F. 350

van Gilst, W.H. 493

van Rooijen, N. 10

Vennemann, P. 54

Vermeer, C. 427

Vinh, A. 143

Vink, A. 244

Virgintino, D. 129

Völker, W. 103

Vosseler, M. 45

Vournakis, J.N. 222
Wadsworth, R.M. 375

Wafai, R. 279

Wallerath, T. 480

Walter, A. 386

Walzog, B. 153

Wang, N. 132

Wang, Q.-S. 469

Wang, Y.-X. 469

Wara, A.K.M.K. 303

Weissen-Plenz, G. 103

Westerweel, J. 54

Westvik, T.S. 251

Weyer, G. 402

Widdop, R.E. 143

Wladimiroff, J.W. 54

Wolff, R.A. 365

Won, K.-J. 259

Wright, C.E. 279

Wu, L.Q. 78

Xu, H. 480

Xu, W. 193

Yadav, V. 469

Yallampalli, C. 33

Yallampalli, U. 33

Yamane, T. 303

Yang, H.H.C. 314

Yang, Z.H. 78

Yassari, R. 402

Yasuda, S. 123

Yasui, S. 233

Ye, L.Y. 78

Yoshida, Y. 303

Yoshimitsu, K. 123

Zandvoort, A. 493

Zani, F. 512

Zhang, W.J. 78

Zhang, Z.-D. 402

Zheng, Y.-M. 469

Zijlstra, F. 493

Zoellner, H. 193 